\title{
Tabularia
}

TABULARIA Sources écrites des mondes normands médiévaux

Autour de Serlon de Bayeux : la poésie normande aux $\mathrm{XI}^{\mathrm{e}}-\mathrm{XII}{ }^{\mathrm{e}}$ siècles | 2017

\section{Raoul Tortaire : mon voyage en Normandie}

Ralph Tortaire: my journey in Normandy

Rodolfo Tortario : Il mio viaggio in Normandia

\section{Pierre Bouet}

\section{OpenEdition}

\section{Journals}

Édition électronique

URL : http://journals.openedition.org/tabularia/2813

DOI : 10.4000/tabularia. 2813

ISSN : 1630-7364

Éditeur :

CRAHAM - Centre Michel de Boüard, Presses universitaires de Caen

Référence électronique

Pierre Bouet, « Raoul Tortaire : mon voyage en Normandie », Tabularia [En ligne], Autour de Serlon de Bayeux : la poésie normande aux Xle-XIIe siècles, mis en ligne le 02 février 2017, consulté le 01 mai 2019. URL : http://journals.openedition.org/tabularia/2813 ; DOI : 10.4000/tabularia.2813 



\section{Raoul Tortaire: mon voyage en Normandie Ralph Tortaire: my journey in Normandy Rodolfo Tortario: Il mio viaggio in Normandia}

Pierre BOUET

Normandie Univ, UNICAEN

pfbouet@free.fr

Résumé:

Raoul Tortaire, moine de l'abbaye de Saint-Benoît-sur-Loire, composa de nombreuses œuvres poétiques. Il rédigea notamment un ensemble de onze épîtres en distiques élégiaques, dont la neuvième est consacrée à un voyage en Normandie. Dans ce poème, il décrit la ville de Caen, son château, son port et son marché, sans parler toutefois des deux abbatiales de La Trinité et de Saint-Étienne, édifiées par Guillaume et Mathilde. Il est le témoin d'un défilé somptueux du roi d'Angleterre, qui se montre au peuple avec une partie de sa ménagerie exotique. Il raconte en détail son périple par le bord de mer entre Caen et Bayeux avec tous les incidents de parcours: il assiste ainsi à une chasse à la baleine qui l'a fortement impressionné. De la ville de Bayeux il ne retient que la cathédrale, dont il décrit les splendeurs intérieures et extérieures. Son aventure se termine dans une auberge où on lui sert en guise de vin un "extrait de pommes acides", qu'il considère comme un vrai poison. Ce poème manifeste avec évidence la culture d'un moine qui connaissait les grands poètes de l'Antiquité et qui, dans son monastère, était chargé d'enseigner l'art d'écrire et l'art de composer des vers.

Daté traditionnellement du règne du roi Henri I ${ }^{\text {er }}$ (1100-1135), peu après l'incendie de la cathédrale en 1105, ce voyage nous a semblé antérieur à cet événement: il s'agit plus vraisemblablement d'une visite de Raoul Tortaire à l'époque où le roi Guillaume le Roux (1087-1100) avait en charge le duché de Normandie, du fait du départ de son frère Robert Courteheuse en Croisade, entre 1096 et 1099.

À la suite de cette étude, nous avons proposé une édition avec traduction du passage de l'épître IX qui concerne les villes de Caen et de Bayeux (vers 137-316).

Mots-clés: Normandie, Caen, Bayeux, baleine, cathédrale, couronne de lumière, pommes, $\mathrm{XI}^{\mathrm{e}}$ siècle, $\mathrm{XII}^{\mathrm{e}}$ siècle

Abstract:

Ralph Tortaire, a monk of the abbey of Saint-Benoit-sur-Loire was the author of many poems. In particular he composed a set of seven epistles in elegiac couplets, the ninth of which is devoted to a journey to Normandy. In this poem he describes the town of Caen, its castle, its port, and its market, but does not mention, the two abbey churches of Saint-Etienne and La Trinité, built by William and Matilda. He witnesses a magnificent procession by the king of England, who appears publicly with some of his menagerie of exotic animals. He gives a detailed account of his journey along the sea coast between Caen and Bayeux, including all the incidents that took place; he was present at a hunt for a whale that impressed him greatly. Of the ton of Bayeux, 
he mentions only the cathedral, whose internal and external magnificence he describes. His journey ends at an inn where he is served a drink described as "an extract of acidic apples" under the pretence that it is wine. He describes it as being truly poisonous. The poem displays the culture and the learning of a monk who knows the work of the great poets of Antiquity, and who, within his monastery was responsible for teaching writing and the composition of verse.

Traditionally dated to the reign of King Henry I (1100-1135), shortly after the cathedral of Bayeux was burnt in 1105, the journey seems to us to have more likely to have taken place earlier. Ralph Tortaire's visit probably took place when King William Rufus (1087-1100) was present in the duchy in one of the years between 1096 and 1099 when he was in charge of it while his brother Robert Curthose was taking part in the First Crusade.

Following this study, we proposed an edition with translation of the passage from the Epistle IX concerning the cities of Caen and Bayeux (circa 137-316).

Keywords: Normandy, Caen, Bayeux, whale, cathedral, crown of light, apples, XI ${ }^{\text {th }}$ century, XII ${ }^{\text {th }}$ century

Riassunto*:

Rodolfo Tortario, monaco dell'abbazia di Saint-Benoît-sur-Loire, è autore di numerose opere poetiche. In particolare, egli ha composto undici epistole in distici elegiaci, la nona delle quali è dedicata ad un viaggio compiuto in Normandia. In questa poesia, Rodolfo descrive la città di Caen, il suo castello, il suo porto e il suo mercato, senza tuttavia parlare delle due abbazie della Trinité e di Saint-Étienne, fatte costruire da Guglielmo e Matilde. Egli è testimone di una sontuosa sfilata del re d'Inghilterra, che si mostra al popolo in compagnia di una parte del suo serraglio esotico. Racconta dettagliatamente il suo viaggio lungo la costa tra Caen e Bayeux con tutti gli incidenti di percorso: egli si trova cosi ad assistere ad una caccia alla balena che l'ha fortemente colpito. Della città di Bayeux egli non parla che della cattedrale, di cui descrive lo splendore interno ed esterno. La sua avventura si conclude in una locanda dove al posto del vino gli viene servito un "estratto di mele acide», che egli considera un vero e proprio veleno. Questo componimento mostra in modo evidente la cultura di un monaco che conosceva i grandi poeti dell'Antichità e che, nel suo monastero, aveva il compito di insegnare l'arte di scrivere e di comporre versi.

Tradizionalmente datato al regno del re Enrico I (1100-1135), poco dopo l'incendio della cattedrale nel 1105, questo viaggio ci è sembrato anteriore a questo avvenimento: si tratta di una visita di Rodolfo Tortario risalente, più verosimilmente, all'epoca in cui il re Guglielmo il Rosso (1087-1100) aveva la reggenza del ducato di Normandia, in seguito alla partenza di suo fratello Roberto II Cosciacorta per la Crociata, tra il 1096 e il 1099.

In seguito a questo studio, abbiamo proposto un'edizione con traduzione del passaggio dell'epistola IX che riguarda le città di Caen e di Bayeux (versi 137-316).

Parole chiave: Normandia, Caen, Bayeux, balena, cattedrale, corona di luce, mele, XI secolo, XII secolo

Raoul Tortaire (ou La Tourte) ${ }^{1}$ est un moine de l'abbaye bénédictine de Fleurysur-Loire. D’après ses propres confidences, il serait né à Gien vers 1060. Il entra au monastère de Saint-Benoît-sur-Loire où il fut formé aux arts libéraux. Selon

* NDLR: Traduction italienne par Laura Vangone, Centre de Recherches Archéologiques et Historiques Anciennes et Médiévales, Normandie Univ, UNICAEN, CNRS, CRAHAM, 14000 Caen, France.

1. Histoire littéraire de la France, vol. X; Dubois, 1824, I; Pluquet, 1829; Certain, 1855; Ogle et SCHULlian, 1933; BAR, 1937. 
une autre confidence, il se serait consacré à l'écriture sur le tard, à l'âge de 45 ans. La date de sa mort est aussi incertaine que celle de sa naissance: on estime qu'il serait décédé peu après 1142, puisqu'il était présent à Cluny au moment de la mort de Pierre Abélard².

\section{Les œuvres de Raoul Tortaire et la $9^{\mathrm{e}}$ épître}

Raoul a été un écrivain prolixe. La communauté de Fleury-sur-Loire le choisit pour continuer le recueil des miracles accomplis par saint Benoît. Ce recueil avait été commencé au IX ${ }^{\mathrm{e}}$ siècle par le moine Adrevald, afin de faire connaître les miracles opérés par saint Benoît à l'abbaye de Fleury et dans les prieurés qui en dépendaient. L'ouvrage fut poursuivi par les moines Aimoin et André. Raoul Tortaire prit le relais de ce dernier, en rédigeant les prodiges qui se seraient produits durant le règne du roi de France, Philippe $\mathrm{I}^{\mathrm{er}}$ (1060-1108). Son successeur, Hugues de Sainte-Marie, commença sa collaboration, en relatant un miracle de l'année 1113. Cette partie du recueil des Miracles de saint Benoît est la seule œuvre en prose de Raoul Tortaire. Il était davantage porté vers la poésie, telle que l'avait pratiquée les auteurs anciens. Il fut d'ailleurs chargé de cet enseignement à l'école du monastère.

Il composa plusieurs ouvrages en vers: un long poème, le De memorabilibus, en neuf livres, qui s'inspire fidèlement des Facta et dicta memorabilia de ValèreMaxime $^{3}$, une adaptation en vers, sous forme de quatrains, de l'ouvrage en prose des Miracula sancti Benedicti, une Passio beati Mauri et onze épîtres en vers. Tous ces poèmes sont composés en distiques élégiaques ou en hexamètres dactyliques, à l'exception d'un Hymne en l'honneur de saint Maur, qui comprend 79 strophes en vers saphiques ${ }^{4}$.

Les Epistulae ad diversos constituent la partie la plus originale et la plus personnelle de la production de Raoul Tortaire ${ }^{5}$. La première épître est adressée à un poète du nom de Garnier Bourdon, dans laquelle l'auteur se plaint de l'absence de mécènes pour venir au secours des poètes et de la poésie. Aujourd'hui, dit-il, Virgile, faute de parchemin et de cire, serait condamné à mourir de faim. La deuxième épître est un éloge de l'amitié et des couples célèbres comme Damon et Pythias, Nisus et Euryale et Ami et Amile: cette épître constitue, d'ailleurs, le plus ancien témoignage de la célèbre Chanson d'Ami et d'Amile. La troisième épître, adressée à un certain Eude, traite du mépris des richesses, auxquelles il convient de préférer la vertu, tandis que la quatrième, adressée au même destinataire, est consacrée au respect des règles de la vie morale. Dans la cinquième épître,

2. Dolveck, 2013 : F. Dolveck, au terme d'un examen minutieux du manuscrit contenant les œuvres de Raoul Tortaire (Vatican, Reg. Lat. 1357 daté du XII e siècle), a attribué à Raoul Tortaire une épitaphe composée en l'honneur de Pierre Abélard, mort en 1142.

3. Bretzigheimer, 2007; Sivo, 2003; ID., 2014.

4. Outre cet Hymnus in honore beati Mauri (Ogle et Schullian, 1933), Raoul Tortaire a composé la Passio beati Mauri comprenant un Prologus et une Oratio en distiques élégiaques de 114 et 112 vers ainsi que deux livres de 397 et 504 hexamètres dactyliques.

5. Ogle et Schullian, 1933. 
il regrette le silence du jeune noble qu'il avait formé à l'art poétique et dont il espérait une amitié fidèle. Il croit expliquer ce silence par le souvenir, que le jeune homme a peut-être gardé, des sévérités que le maitre lui infligeait pour le bien de sa formation. Dans la septième épître, dont le destinataire est Galon, évêque de Paris (1105-1116), Raoul évoque une expédition conduite par Bohémond peu après la première croisade. La neuvième épître raconte le voyage que le poète effectua en Normandie en compagnie de son ami Pierre ${ }^{6}$.

Cette épître de 334 vers en distiques élégiaques, connue par un seul manuscrit (Vatican, Reg., 1357), commence par des considérations sur sa fidélité envers son correspondant, un certain Robert: il s'excuse de n'avoir pas répondu à sa dernière lettre en le justifiant par la lourde charge de ses obligations. Il se félicite de n'être ni l'insaisissable Protée, ni l'astucieux Ulysse dont la perfidie a causé la mort de nombreuses personnes. Il l'invite à faire comme lui, à prendre du repos et à se détendre. Le marin, qui rentre exténué au port, ne repart jamais aussitôt en mer. De même les rois, accablés de soucis, savent les oublier en allant à la chasse. Raoul et son ami Pierre visitent Caen, puis se rendent à Bayeux en passant par la côte. Le récit de ce périple normand présente de nombreuses informations sur la Normandie de la fin du $\mathrm{XI}^{\mathrm{e}}$ siècle.

\section{La Normandie de Raoul Tortaire}

Cette épître de Raoul Tortaire est un témoignage extrêmement précieux pour notre connaissance des villes de Caen et de Bayeux au début du Xlle siècle, même si nous regrettons souvent que l'auteur n'ait pas été plus prolixe.

Raoul se plaît d'abord à décrire le site de la ville de Caen (situm Cadomi describere, v. 213), dont, dit-il, personne n’a jamais parlé avant lui. En ce début du $\mathrm{XII}^{\mathrm{e}}$ siècle, Caen vient tout juste d'émerger de l'ombre, à la suite de la fondation entre 1059 et 1066, des deux abbayes caennaises, l'Abbaye-aux-Hommes à l'ouest et l'Abbaye-aux-Dames à l'est, dont le moine de Fleury ne nous parle même pas $^{7}$. Auparavant Caen n'était présentée dans les chartes que comme un burgus ou une villa. Ainsi, en 1025, dans une charte de Fécamp, Caen était qualifiée de urgus $^{8}$; elle faisait, en outre, partie du douaire d'Adèle, l'épouse du duc Richard III, constitué en 1026-10279 :

Et dans le comté de Bayeux je donne le domaine qui s'appelle Caen sur l'Orne avec églises, vignes, prés, moulins, marché (foro), tonlieu, port et toutes les dépendances.

6. La sixième épître (Ad Sincopum) n'est qu'un conte grossier, tandis que la huitième (Ad Burdonem) est en grande partie illisible.

7. Sur la ville de Caen au XIe siècle, voir Neveux, 1997, I; DÉsert, 1981; JeAn-Marie, 2000.

8. Fauroux, 1961, n 34, p. 130 (charte du duc Richard II pour Fécamp, août 1025): Do etiam decimas telonei de burgo qui dicitur Cadumus.

9. Ibid., $\mathrm{n}^{\circ}$ 58, p. 182 (douaire d'Adèle): Et in comitatu Baiocensi, concedo villam que dicitur Cathim super fluvium Olne circumquaque cum ecclesiis, vineis, pratis, molendinis, cum foro, theloneo et portu et omnibus appenditiis suis. 
Nous comprenons, à la lecture de Raoul Tortaire, que Caen est devenue, quelque 50 ans après, une grande ville, où le roi d'Angleterre tient à parader.

La Muse bavarde brûle de décrire le site de Caen, dont il n'a été question nulle part ailleurs. Vois le château situé sur la rive, près du fleuve, à l'endroit le plus élevé de la colline. Au sud, des prés verts de faible largeur s'étendent sur près de trois milles, tout près du fleuve. Le port abrite des navires imposants, que la mer lui envoie, là où le fleuve a du mal à s'écouler en raison du reflux de la mer dans l'estuaire. La terre est riche en moissons, mais pauvre en forêts épaisses. Font défaut la noix gauloise, la vigne, le figuier et l'olivier. Telle est la cité que l'Angleterre, une île entourée par l'océan occidental, enrichit de diverses marchandises ${ }^{10}$.

Raoul parle en premier du château (castrum) situé à l'endroit le plus élevé de la colline et sur les bords de l'Orne (In ripa positum, fluvii latus, aspice castrum/Ad Boream, collis ediciore loco, v. 215-216). Au sud, en réalité entre les méandres de l'Odon et l'Orne s'étendent des prés verdoyants sur près de trois milles (Prata virent latus ad Nothium, v. 217). À l'ouest, se trouve le port, où viennent à quai d'imposants navires (grandes naves) qui apportent pour l'essentiel des marchandises venues d'Angleterre, une île entourée par la mer, tient à préciser Raoul (Insula diversis quam mercibus Anglia ditat, v. 223). Il est surpris par la marée qui remonte jusque dans le port et qui empêche les eaux du fleuve de s'écouler normalement. Il constate, enfin, que la région caennaise (que nous appelons la Plaine de Caen) produit uniquement des céréales et qu'elle est dépourvue de forêt. Il ajoute que le pays ne connaît ni l'olivier, ni le figuier, ni la vigne, ni la noix gauloise.

À deux reprises, Raoul déplore que la Normandie ne connaisse pas la vigne. Bacchus, le fils de Sémélé, qui a pourtant réussi à soumettre «les peuples vipérins nés des dents des dragons ainsi que les noirs Indiens», n'est pas encore parvenu à terrasser «les inflexibles Normands» (rigidos Normannos) ${ }^{11}$. Peut-être ignore-t-il que la vigne était cultivée dans de nombreux endroits du duché. Il y avait même des vignes sur les pentes du château à Caen. Ou plutôt Raoul le savait, mais estimait que ce breuvage ne méritait pas le nom de vin.

Raoul a été séduit et impressionné par le dynamisme de la cité. S’il évoque brièvement la beauté architecturale des édifices, qui « resplendissent comme s'ils étaient en marbre de Paros" (Quae tanquam Pario marmore tota nitent, v. 137), il décrit avec soin la place du marché où arrivent de partout marchands et acheteurs ${ }^{12}$. Nous sommes donc un lundi, qui est au Moyen Âge, le jour du marché à Caen. Il compare la cité à une ruche où, comme dans le monde des abeilles, chacun remplit une fonction essentielle dans une grande harmonie. Tous ses sens sont séduits lors de sa déambulation parmi les étals des marchands. Il voit, il sent, il touche, il entend, malheureusement il ne peut goûter, faute

10. Vers 213-225.

11. Vers $307-312$.

12. Vers 136-142. 
d'argent (Cum delata foro venalia tanta viderem/non essent nummi quod mihi, dirigui, v. 161-162). Dans une profusion incroyable, on propose, ici cumin, cannelle, poivre, miel, cire, encens (Cinnama, thura, piper, pomum, mel, cera, ciminum, v. 151), là des toiles de lin au fin tissage (tenuis texturae copia lini, v. 149) et des soies fines et souples (multiplicis fili serica mollicies, v. 150). Tout proche se tient le marché aux bestiaux où se mêlent ovins, sangliers, chevaux dressés et chevaux non dressés (indomitis mixti cornipedes domiti, v. 156). Les clients pouvaient aussi bien acheter leur viande à l'étal du boucher qui exposait des carcasses de bœufs évidés de leurs viscères (nudatisque boum tergora visceribus, v. 154) et des aliments de toutes sortes en même temps que des boissons. Dans cette cohue se mêlaient des gens aux habits différents; quant aux femmes on les reconnaissait à leurs hautes coiffures en pointe (Vidi disparium facies habitusque virorum / Feminei sexus carbaseos apices, v. 159-160).

Une chance rarissime s'est offerte à Raoul Tortaire et à son compagnon durant leur séjour dans la ville de Caen. Ils ont assisté à la parade du roi d'Angleterre, qui a traversé la cité avec son escorte et son équipage. Le roi portait un vêtement en lin de couleur pourpre rehaussé d'or (Purpura cum bisso regem vestibat et auro, v. 199). L'or des boucliers des chevaliers qui accompagnaient le souverain renvoyait les rayons du soleil et les spectateurs étaient frappés par ces éclairs de lumière. Mais le roi n'était pas venu seul: il avait sorti de son parc quelques animaux exotiques qui participaient à la parade royale pour le plus grand bonheur d'une foule curieuse, fascinée par un tel spectacle. En tête s'avançait un jeune lion aux rugissements effrayants, qui épouvantait la foule (Terrebat vulgus rictibus horrisonis, v. 168). Ensuite un léopard aux taches sombres s'agrippait à la crinière d'un cheval terrorisé (Hinc maculis leopardus equo pulcherrimus atris/Colla vehebatur, nexibus implicitus, v. 171-172). Un lynx au regard perçant ouvrait une gueule terrifiante (ore trucem lincem, v. 176). Suivait un dromadaire avec sa bosse au milieu du dos (Lubrica gibbosum ductabat turba camelum, v. 183), un animal qui vit deux cents ans et qui est, chose surprenante, capable de rester sans boire pendant quatre jours (Quadriduam tolerare sitim, v. 189). Une autruche avec son long cou et ses pattes démesurées fermait la marche de ce cortège royal (...structio.../Collo procero, cruribus inmodicis, v. 193-194).

On peut s'interroger sur les raisons qui conduisent les princes, les rois et les empereurs à se présenter à leurs sujets en compagnie d'une partie de leur ménagerie. Bien des sources nous apprennent que, depuis l'époque carolingienne, on se plaisait à posséder dans son parc des animaux exotiques. Déjà, Geoffroy de Montbray, l'évêque de Coutances, qui était allé en Italie du sud quêter auprès de son paroissien, Robert Guiscard, pour avoir l'argent nécessaire à la restauration de sa cathédrale et de son palais épiscopal, avait créé un immense parc de plus de cent hectares, dans lequel il entretenait des animaux domestiques et sauvages ${ }^{13}$. Selon Widukind de Corbie, l'empereur Otton pensait étendre son

13. Gallia christiana, vol. XI, Instrumenta, col. 219: ibique parcum opulentissimum cervis et apris, tauris et vaccis et equis constituit. 
pouvoir impérial sur l'Afrique et l'Asie en possédant des animaux de ces pays ${ }^{14}$. La présence d'un animal exotique est souvent le signe que le prince est reconnu par ses pairs et que sa renommée dépasse les frontières. L'animal exotique est très souvent offert par un prince lointain, comme l'éléphant que s'était vu offrir Charlemagne par le calife de Bagdad, Haroun al Rachid ${ }^{15}$. À l'inverse, abattre la ménagerie de son adversaire en faisant détruire ses parcs et ses jardins équivaut à l'anéantissement symbolique de son pouvoir. C'est ainsi que procéda l'empereur germanique, Henri VI, quand il s'empara de Palerme en 1194 et qu'il s'appropria le pouvoir détenu par les rois normands: il fit raser les jardins de la Conque d'or et tuer tous les animaux exotiques ${ }^{16}$. Des animaux comme le lion, le léopard, le lynx, l'autruche sont perçus principalement dans leur valeur symbolique: c'est justement ce que montrent les premiers bestiaires, comme celui de Philippe de Thaon dédié à l'épouse du roi Henri $\mathrm{I}^{\mathrm{er}}$ Beauclerc ${ }^{17}$. Ils jouent donc un rôle capital dans la mise en scène du pouvoir et dans l'image que le souverain veut imposer dans l'esprit de ses sujets ${ }^{18}$.

La ville de Bayeux est le terme du voyage de Raoul et de son ami Pierre, sans que l'auteur ne nous donne les raisons de ce long périple ${ }^{19}$. Il consacre l'essentiel de son propos à la description de la cathédrale de Bayeux.

De là, je vais à Bayeux, où j'ai vu des édifices somptueux, les hautes tours d'une église honorable, qui parfaitement achevée resplendit à l'intérieur par ses pierres parfaitement polies et à l'extérieur par ses statues. Elle s'enrichit d'une abondance d'objets de divers métaux ainsi que d'une toile de lin, de couleur pourpre ou vermeil. Des chaînes en fer, fixées tout en haut à la clef de voûtes par de solides attaches, supportent une couronne d'argent. Celle-ci est entièrement recouverte d'or étincelant et le forgeron a disposé tout autour des édicules surmontés de tours. Elle n'a pas, à mon avis, son pareil en poids et trouve difficilement place entre les deux murs de l'église.

Ce qu'il aperçoit en premier ce sont les tours, vraisemblablement les deux tours de façade et la tour lanterne de la croisée du transept (turres excelsas aedis honorificae, v. 292). Il est particulièrement sensible à la qualité des pierres parfaitement polies à l'intérieur de l'édifice, ce qui doit correspondre en réalité au décor peint en forme de grand appareil sur un enduit masquant les matériaux (Intus quae saxo pulchre perfecta polito, v. 293). Il admire tout autant les statues qui ornent l'extérieur du monument, ce qui laisse supposer que les trois portails romans abritaient de nombreuses statues, comme on peut le voir aujourd'hui encore sur des façades romanes (Exterius sculptis fulget imaginibus, v. 294).

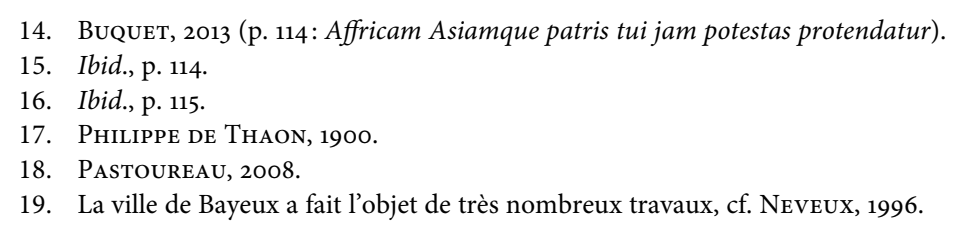


Dans la cathédrale, un objet le fascine c'est la couronne de lumière que l'évêque Odon fit faire pour sa cathédrale et qui disparut lors des guerres de religion en 1562: Ferrea sustentant argenti vincla coronam / Alta quae dura sunt clave fixa sude, / Tota superficies auro vestita renidet/Cinxit turritis quam faber aediculis (v. 297-300).

La présence de cette couronne de lumière, sans la moindre allusion à des traces d'incendie ou de destruction à l'intérieur de la cathédrale, fait difficulté, si l'on a en mémoire les vers de Serlon, décrivant l'incendie de la cathédrale en $1105^{20}$. Ces lignes de Raoul Tortaire nous incitent à envisager plusieurs hypothèses sur la datation de ce voyage. Raoul a pu effectuer son périple avant 1105, avant que le roi Henri I ${ }^{\text {er }}$ 'ait envahi la Normandie et assiégé la ville de Bayeux. Mais alors comment expliquer la présence du roi d'Angleterre avec tout son cortège à Caen, alors qu'il est en conflit avec son frère, le duc Robert Courteheuse $^{21}$. Raoul a pu aussi bien entreprendre ce voyage après 1105, mais alors, cela laisse supposer, comme l'a déjà suggéré Frédéric Épaud dans sa thèse sur les charpentes, que la cathédrale n'avait guère souffert du siège et de l'incendie de la ville de Bayeux ${ }^{22}$. On comprend mal comment une couronne de lumière, fixée à la voûte, aurait pu résister à un incendie, qui aurait embrasé tout l'édifice et principalement les charpentes, comme le relate Serlon. On pourrait alors imaginer que la rancœur du doyen Serlon l'ait incité à exagérer l'importance des destructions. Ce qui est sûr, c'est que les restaurations de 1856, les études archéologiques de Jean Vallery-Radot et les expertises de Frédéric Épaud montrent que les murs du chœur et le haut des piles romanes de la tour de croisée étaient intacts sous le revêtement gothique du XIII ${ }^{e}$ siècle $^{23}$. Frédéric Épaud s'appuie sur la datation des frettes crénelées des cinq premières travées de la nef, effectuée par M. Baylé, pour supposer que l'incendie n'aurait touché que la partie occidentale de la nef et les tours de façade ${ }^{24}$. Reste une troisième hypothèse: Raoul aurait effectué son voyage avant 1100, au temps où Robert Courteheuse, qui était le duc légitime, était parti en croisade et avait confié la gestion des affaires du duché à son frère Guillaume le Roux, roi d'Angleterre (1087-110o). Dès lors, se trouvent conciliées, à la fois, la parade royale dans la ville de Caen et la description de la cathédrale de Bayeux dans toute sa splendeur ${ }^{25}$. La parade serait celle, non

20. SERLON, De capta Bajocensium civitate, 1872: Hac fuit usta die sacra Virginis aula Mariae, / templaque bis quina simili periere ruina («En ce jour l'église de la Vierge Marie brûla et dix églises tombèrent semblablement en ruine»).

21. Sur l'histoire du royaume anglo-normand entre 1087 et 1135, voir GreEN, 2006, BARLOW, 2008 et AIRD, 2008.

22. ÉPAud, 2007, p. 7

23. Dion et LASVignes, 1861; VAllery-RAdot, 1958, p. 13.

24. BAYLÉ, 1997.

25. Lors d'une conférence présentée au Château de Caen, le 3 septembre 2016, Edward Impey a démontré que la salle de l'Échiquier ne datait pas du règne de Henri I ${ }^{\text {er }}$ Beauclerc (vers 1122), mais de celui de son frère Guillaume le Roux (1087-1100). Guillaume le Roux gouverna, en effet, la Normandie durant l'absence de Robert Courteheuse, parti à la Croisade de 1096 à 1099. Le roi se montra très actif durant cette période aussi bien à Caen qu'à Rouen: la parade racontée par Raoul Tortaire trouve sa pleine justification durant la période 1096-1099. 
pas de Henri I ${ }^{\text {er }}$ Beauclerc, mais de son frère, Guillaume le Roux, tout aussi passionné que lui de chasse et d'animaux exotiques, qu'il aurait effectuée entre septembre 1096 et décembre $1099^{26}$.

L'Épître de Raoul Tortaire nous donne aussi des informations sur ses incidents de voyage et sur des événements dont il a été le témoin. Il raconte la mort de son cheval, alors qu'il traverse un petit ruisseau en terrain marécageux entre Caen et la mer. Comme le cheval était assoiffé - on avait empêché les montures de boire dans les eaux de l'Orne, à Caen, en raison de la pollution: Impotabilis est nam fluvius Cadomi (v. 226) -, au lieu de boire sur la berge, comme le font les autres chevaux, saute au milieu du ruisseau et s'y enfonce jusqu'au poitrail. Quand il dégage ses jambes avant, les jambes arrière s'enfoncent davantage. Malgré les tentatives du cavalier qui est, lui aussi, en grand danger, l'animal s'enlise et s'écroule mort. Raoul qui n'a pas eu le temps de dégager ses pieds des étriers, tombe avec sa monture et est sauvé de justesse par ses compagnons.

Le voyage se poursuit le long de la côte. Raoul et ses compagnons sont hébergés dans des villae en bord de mer où ils dégustent toutes sortes de poissons aux saveurs différentes. Raoul se plait à se promener dans la mer, jambes nues, dans les mares laissées par la marée basse. C'est dans ces «plates» que les marins en hiver font échouer les baleines avant de les tuer. Il nous raconte une chasse à la baleine à laquelle il a assisté (v. 279-290 ${ }^{27}$. Il évoque le matériel qui est soigneusement préparé par les marins avant la chasse. Ils ont tissé des filets avec du chanvre enroulé, ils triplent les câbles pour que ceux-ci ne se rompent sous le poids des baleines, ils ont aiguisé les harpons, dont certains étaient à triple pointe. Ces expéditions d'hiver s'effectuaient toujours sur une mer déchaînée. Au danger de la mer s'ajoutait celui de voir l'une ou l'autre embarcation être envoyée au fond par un puissant coup de queue du monstre. Il a été également impressionné par les jets d'eau immenses que projetaient ces animaux par leurs $«$ narines ${ }^{28}$. L'expédition dont il a été le témoin s'est mal terminée, car la baleine a réussi à rompre le filet. Or, nous savons que la chasse à la baleine était une activité essentielle en Normandie à cette époque et qu'elle se pratiquait dans de nombreux ports ${ }^{29}$.

26. On aurait pu estimer que cette parade avait eu lieu en 1091: le roi Guillaume le Roux vint, en effet, à Caen se réconcilier avec son frère Robert Courteheuse (traité de Caen). Mais on comprendrait mal que cette parade dans la ville se soit faite sans la présence conjointe du duc de Normandie.

27. Cf. vers 279 à 290; sur la chasse à la baleine, voir Musset, 1997, notamment p. 312 : Lucien Musset considère le type de chasse décrit par Raoul Tortaire «à cry et à hue» (selon une expression du XVI ${ }^{e}$ siècle), comme une technique d'origine scandinave encore en usage aux îles Féroé. Cf. également Lestocquoy, 1948 et LebecQ, 1997, qui ne parlent pas de Raoul Tortaire: ils évoquent l'importance de cette chasse depuis le haut Moyen Âge et l'existence de puissantes corporations, comme celle des Whalmans à Caen.

28. Raoul Tortaire parle de nares («narines») pour qualifier «les évents» des baleines. L'évent est, en effet, un orifice situé sur la tête de l'animal qui lui permet d'expulser l'air inspiré, mêlé à des gouttelettes d'eau. Les cétacés à fanons possèdent deux évents, tandis que les cétacés à dents n'en ont qu'un.

29. Lucien Musset (Musset, 1964) évoque les lieux où se trouvaient les communautés de baleiniers: La Saire, La Hougue, Réville, Lestre, Quinéville, Saint-Marcouf, Héauville dans le Cotentin; Port-en-Bessin, Saint-Côme-de-Fresné, Dives-sur-Mer, Cabourg, Caen, Quillebeuf, Le Tréport sur les côtes du Calvados et de la Seine-Maritime. 
Les deux derniers épisodes qui couronnent ce voyage se passent à Bayeux. Poussé par la soif, il entre dans une modeste demeure et commande du vin. On lui apporte une boisson qui est un extrait de "pommes acides» (At sucus pomis datus est extortus acerbis, v. 305). Certes le mot «cidre» (formé à partir de sicera en latin populaire) n'est pas présent, mais l'expression sucus extortus pomis acerbis ne laisse aucun doute sur le genre de boisson qui déplut au palais de Raoul, habitué aux vins de la Loire. Il feint de boire après y avoir goûté, s'essuie les lèvres comme s'il était satisfait et appelle le garçon pour lui demander quelle sorte de poison il lui avait apporté (Cur propinasti, serve, venena?, v. 316). Il remarque une grive dans une cage qui a la faculté d'imiter les paroles humaines: elle ne sait que répéter sans cesse le nom de la servante, «Va-t-en dehors Murion» ou «Viens ici Murion!».

\section{La poésie de Raoul Tortaire}

On ne peut lire cette épître de Raoul Tortaire sans songer immédiatement à la satire V d'Horace, relatant son voyage de Rome à Brindes. Cependant, une comparaison minutieuse des deux œuvres livre son verdict: elles n'ont aucun point commun. Horace raconte avec beaucoup d'esprit les petits incidents qui ont émaillé les différentes étapes de son voyage, en leur conférant une grande importance: moustiques agressifs, grenouilles bavardes, ophtalmie, gastrites empêchent le poète et de dormir et d'apprécier les mets de ses hôtes. Le poète s'attarde longuement sur l'altercation entre deux bouffons à Caudium, sur l'incendie que provoque un cuisinier maladroit en retournant des grives sur un feu, sur la fumée incommodante et suffocante dans la demeure où il est hébergé, sur le pain dur comme la pierre des boulangers de Canusium. Horace ne s'intéresse ni aux paysages de la Campanie et de l'Apulie qu'il traverse, ni aux bourgs et cités où il est reçu. Le poète fait son miel des péripéties sans importance et des détails pittoresques de son aventure. Tout cela est conforme, d'ailleurs, au genre satirique qu'Horace a adopté.

L'épître de Raoul Tortaire procède d'une tout autre intention. Le poète ne s'interdit pas, toutefois, de nous conter quelques mésaventures qui auraient sans doute eu leur place dans la satire d'Horace, comme la promenade en bord de mer où le moine de Fleury ramasse des galets, l'enlisement de son cheval dans une rivière marécageuse et la scène du cabaret bayeusain où l'on sert aux clients des boissons infâmes Pour le reste, l'épître de Raoul Tortaire se propose de faire connaître aux lecteurs la géographie de la ville de Caen avec son château, son port, son marché et ses prairies verdoyantes (qui existent toujours). Il consacre quelque cinquante vers à l'entrée solennelle du roi d'Angleterre dans la cité caennaise avec son escorte et quelques animaux de sa ménagerie. La cathédrale de Bayeux l'impressionne, aussi en donne-t-il une description qui, en dépit de sa brièveté, en montre la somptuosité: les hautes tours, les statues qui ornent l'extérieur de l'édifice, la couronne de lumière offerte par Odon. La présence de tentures en lin, teintes en pourpre et en vermeil, qui ornent les murs intérieurs de la cathédrale rend vraisemblable l'exposition de la Broderie de Bayeux dès cette époque. 
Il n'est donc pas surprenant de ne rencontrer dans l'épître de Raoul Tortaire aucun terme, aucune image qui témoigne d'une quelconque imitation de la satire d'Horace. Le relevé des expressions empruntées aux auteurs de l'Antiquité classique manifeste une solide culture poétique où Virgile et Ovide occupent une place éminente ${ }^{30}$. Raoul a le souci d'intégrer à sa métrique et à son propos les réminiscences antiques, comme le montrent ces deux exemples:

Raoul Tortaire, 154: Nudatisque boum tergora visceribus

Virgile, Énéide, 1, 211 : Tergora diripiunt costis et viscera nudant

Raoul Tortaire, 144: A fluvio sumptas pars quoque gestat aquas

Ovide, Les Amours, 2, 19: E magno flumine sumat aquam

Cette présence des Anciens est perceptible, lorsque Raoul Tortaire cherche à conférer à sa poésie une tonalité épique. Ainsi, quand il évoque l'escorte royale, il compare les reflets du soleil sur l'or des boucliers des chevaliers aux éclairs qui lors des tempêtes frappent et illuminent la nature. Dans ces lignes le lexique est emprunté à la fois à Virgile et à Lucrèce.

Raoul Tortaire, 203: Solis inaurati clypei fulgore micabant

Virgile, Énéide, 9, 733: Clipeoque micantia fulmina mittit

Raoul Tortaire, 211-212: Non aliter quam cum tonitrus quatit omnia saevus Concava riparum terraque mota tremit.

Lucrèce, 6, 96: Principio tonitru quatiuntur caerula caeli

Virgile, Géorgiques, 1, 330: ... quo maxima motu/terra tremit

On retrouve cette même tonalité épique dans le récit de la mort du cheval dans le marécage

Raoul Tortaire, 247 : Ilia quadrupedis rumpuntur agone paludis

Virgile, Bucoliques, 7, 26: invidia rumpantur ut ilia Codro

Raoul Tortaire, 249: Qui cursu rapido rapidos praecurreret Euros

Virgile, Énéide, 12: qui candore nives anteirent cursibus auras

30. Nous reprenons ici l'étude des sources littéraires effectuées par notre maître, Francis Bar, cf. BAR, 1937, p. 207-223. 
ou dans celui de la chasse à la baleine:

Raoul Tortaire, 284: Sulcat et aequoreum panda carina vadum

Virgile, Énéide, 10, 197: ... et longa sulcat maria alta carina

Ovide, Héroïdes, 16, 112 : panda carina

Lorsque Raoul Tortaire énumère les animaux exotiques qui participent à la parade royale, il présente pour chacun d'eux des particularités physiques, que lui ont appris, directement et plus vraisemblablement indirectement, Pline l'Ancien ou Solin et Isidore de Séville, comme l'a déjà montré Francis Bar ${ }^{31}$.

Le jeune lion qui terrorise par ses rugissements les habitants de Caen est présenté comme un animal doux et bienveillant envers les malheureux, comme l'affirmait Pline:

Raoul Tortaire, 169-170: Exulibus, miseris, prostratis ac spoliatis Commodus et mitis dicitur esse leo.

Pline, 8, 19, 1: Leoni tantum ex feris clementia in supplices; prostratis parcit

C'est Isidore de Séville qui affirme que le léopard est né du croisement d'une lionne avec un pard (panthère mâle). Pline et Solin considèrent eux aussi que le léopard est un bâtard du pard et de la lionne, mais ils le nomment toujours leo:

Raoul Tortaire, 173: Hunc creat in torva pardi genitura leaena

Isidore, Étymologies, 12, 2, 52 : Leopardus ex adulterio leaeneae et pardi nascitur

Quant au lynx à la vue perçante, c'est Pline qui est le premier à nous le faire savoir:

Raoul Tortaire, 179-180: Denique sic visus acies transverberat ejus Obvia quaeque sibi vix quid ut officiat

Pline, 28, 32, 1 : Peregrinae sunt et lynces, quae clarissime omnium quadripedum cernunt.

Pline et Solin ont été les premiers à affirmer que le chameau vivait cent ans, qu'il pouvait rester quatre jours sans boire, qu'il devenait un transporteur plus puissant si on le castrait, que les femelles étaient très agressives et que le chameau détestait les chevaux.

31. Ibid.

URL: http://tabularia.revues.org/2813 | DOI: 10.4000/tabularia.2813 
Raoul Tortaire, 184: Quem faleris coopertum oppido vitat equus

Solin, 49, 10 : oderunt equinum genus.

Pline, 8, 26: odium adversus equos gerunt naturale

Raoul Tortaire, 188: Bis centum fertur vivere solstitiis

Solin, 49, 12: Durant in annos centum

Raoul Tortaire, 189: Quadriduam tolerare sitim

Solin, 49,10 : sitim etiam in quadriduum tolerant

Raoul Tortaire, 191: Fasci tollendo melior sit teste reciso

Pline, 49, 10: fortiores ita fiunt coitu negato

Raoul Tortaire, 192: Martia femineis bella placent gregibus

Solin, 49, 10: ... ad bella feminae praeparantur

Pour l'autruche, l'autorité est Isidore, qui affirme que l'autruche a des ailes qui ne lui permettent pas de quitter le sol et qu'elle ne couve pas ses œufs qu'elle abandonne dans la terre.

Raoul Tortaire, 195: Qui sua non fovet ova, sed aggere pulveris abdit

Isidore, Étymologies, 12, 7, 91 : Ova sua fovere neglegit, sed projecta tantummodo fotu pulveris animantur.

Raoul Tortaire, 197: Sublevat a terra quem nequaquam sua pluma

Isidore, Étymologies, 12, 7, 91 : tamen de terra altius non elevatur

\section{Conclusion}

La comparaison du texte de Raoul Tortaire avec ces références aux auteurs anciens montre, me semble-t-il, que le moine de Fleury-sur-Loire n'a sans doute pas consulté les ouvrages des auteurs dont nous venons de parler, mais qu'il a plutôt composé sa poésie à partir soit de notes personnelles qu'il s'était constituées quand il enseignait la poésie à l'école de son abbaye, soit d'ouvrages de compilation comme les florilèges, les excerpta qui figuraient dans toutes les bibliothèques médiévales, voire des traités comme le Physiologus. Toutefois il serait téméraire d'affirmer qu'il n'ait pas consulté les ouvrages de Pline l'Ancien et de Solin ainsi que les Étymologies d'Isidore de Séville qui figuraient, comme les œuvres d'Ovide et de Virgile, dans les programmes scolaires du trivium. 
Cette présentation de l'épître 9 de Raoul Tortaire méritait d'être faite pour deux raisons. Elle est d'un intérêt majeur pour notre connaissance de la Normandie à la fin du XI et au début du XII e siècle: rares sont, en effet, les textes en proses et les poésies qui décrivent des bourgs, des cités et des monuments normands. Raoul Tortaire, en outre, s'intéresse à la vie des gens, à leurs coutumes et leur allure. Il montre la richesse d'un duché qui fournit en abondance les fruits de la terre et où arrivent de partout les produits exotiques. Vivant au cœur du royaume de France il découvre la mer, ses séductions et ses dangers. Il eut la chance d'assister pendant son court séjour à une chasse à la baleine, qui mit aux prises des marins audacieux avec un monstre effrayant.

Il eut aussi le privilège d'assister à une parade du roi d'Angleterre dans la ville de Caen. On avait pensé jusqu'alors qu'il s'agissait d'un défilé du roi Henri I ${ }^{\text {er }}$ après sa victoire obtenue à Tinchebray sur son frère Robert Courteheuse en 1106. Mais, une telle hypothèse ne saurait cadrer avec la description de la cathédrale de Bayeux dans toute sa splendeur originelle. Le roi qui parade n'est pas Henri Beauclerc, mais son frère Guillaume le Roux (1087-110o) qui eut en charge le duché durant l'absence du duc Robert Courteheuse, parti en croisade de 1096 à 1099. Cette exhibition de la majesté royale fait partie de l'exercice du pouvoir: le roi doit se montrer à son peuple et à ses dépendants pour renforcer les liens personnels et donner une image idéale de sa grandeur et de sa puissance. Le défilé d'une partie de sa ménagerie exotique contribue à faire du roi un souverain éminent, reconnu par les autres rois étrangers.

L'autre intérêt de ce texte est de constituer un contrepoint aux poésies de Serlon de Bayeux. Cette épître de Raoul constituait, en effet, un argument pour les historiens de l'art qui estimaient que la cathédrale de Bayeux avait peu souffert de l'incendie de 1105. On peut toutefois regretter que le moine de Fleury ait été plus sensible aux malheurs de son cheval enlisé dans les marécages de Caen qu'aux séductions des deux abbayes caennaises dont les façades venaient sans doute d'être achevées.

\section{Bibliographie}

\section{Sources}

Gallia Christiana in provincias ecclesiasticas distributa, t. XI, Paris, Imprimerie royale, 1759, p. 353-362.

Pluquet, Frédéric, Essai historique sur la ville de Bayeux et son arrondissement, Caen, Chalopin, 1829 (édition et traduction de la partie de l'épître IX qui concerne la ville de Bayeux, p. 159-165).

Ogle, Marbury B. et Schullian, Dorothy M., Rodulfi Tortarii Carmina (Papers and Monographs of the American Academy in Rome, VIII), Rome, 1933 (Epistula IX, p. 319-330).

Philippe de Thaon, Bestiaire, éd. E. Walberg, Lund - Paris, Möller - Welter, 1900. 
SERLON, "De capta Bajocensium civitate», in The Anglo-Latin satirical poets and epigrammatists of the twelfth century, Thomas WRIGHT (éd.) vol. II, Londres, 1872, p. 241-251.

\section{Études}

AIRD, William H., Robert Curthose, Duke of Normandy (c. 1050-1134), Woodbridge, Boydell Press, 2008.

BAR, Francis, Les épîtres latines de Raoul Le Tourtier. Études des sources: La légende d'Ami et Amile, Paris, Droz, 1937.

Barlow, Frank, William Rufus, New York, Yale University Press, 2008.

BAYLÉ, Maylis, "La sculpture du XII siècle à Bayeux», in Chapitres et Cathédrales en Normandie, Sylvette Lemagnen (éd.), Actes du XXXI ${ }^{e}$ congrès des sociétés historiques et archéologiques de Normandie, Caen, Musée de Normandie, 1997, p. 461-466.

Bretzigheimer, Gerlinde, «Die De Memorabilibus libri IX des Rodulfus Tortarius. Eine hochmittelalterliche Versifikation der Facta et Dicta memorabilia des Valerius Maximus», in Peter Stотz (dir.), Unter Mitarbeit von Philipp Roelli, Dichten als Stoff-Vermittlung. Formen, Ziele, Wirkungen. Beiträge zur Praxis der Versifikation lateinischer Texte im Mittelalter, Zurich, Chronos, 2008, p. 231-246.

Buquet, Thierry, "Les animaux exotiques dans les ménageries médiévales", in Fabuleuses histoires des bêtes et des hommes, Jacques Toussaint (dir.), Namur, Tréma - Société archéologique de Namur, 2013, p. 97-121.

Certain, Eugène (de), «Raoul Tortaire», Bibliothèque de l'École des Chartes, 16, 1855, p. 489-521 (avec édition du texte latin du vers 140-334).

DÉsert, Gabriel (dir.), Histoire de Caen, Toulouse, Privat, 1981, p. 23-35.

Dion, Henri (de) et LAsvignes, Louis, Reprise en sous-œuvre de la tour centrale de la cathédrale de Bayeux, sous la direction de Louis Flachat, Paris, A. Morel et $\mathrm{C}^{\mathrm{ie}}$, 1861.

Dolveck, Franz, «Un Fleurisien à Cluny: la vie et l'œuvre de Raoul Tortaire», Filologia Mediolatina, 20, 2013, p. 277-308.

Dubois, Louis, Archives de la Normandie, Caen, Mancel, 1824 (I, p. 209 sqq.).

Épaud, Frédéric, Les charpentes de la cathédrale Notre-Dame de Bayeux, Caen, Publications du CRAHM, 2007.

Fauroux, Marie, Recueil des actes des ducs de Normandie de 911 à 1066, Caen, Caron, 1961.

Green, Judith, Henry I. King of England and Duke of Normandy, Cambridge, Cambridge University Press, 2006.

Histoire littéraire de la France, tome X (qui comprend la suite du Douzième siècle de l'Église jusqu'à l'an 1124), Paris, Victor Palmé, 1868, p. 85-94.

Jean-Marie, Laurence, Caen aux XI et XII siècles. Espace urbain, pouvoirs et société, Caen, La Mandragore, 2000. 
LEBECQ, Stéphane, «Scènes de chasse aux mammifères marins dans les mers du Nord, $\mathrm{VI}^{\mathrm{e}}$-XII ${ }^{\mathrm{e}}$ siècle», in Milieux naturels et espaces sociaux, Élizabeth MorNET et Franco Morenzioni (dir.), Études offertes à Robert Delort, Paris, Publications de la Sorbonne, 1997, p. 241-254.

Lestocquoy, Jean, «Baleine et ravitaillement au Moyen Âge», Revue du Nord, 30, p. 39-43.

Musset, Lucien, «Quelques notes sur les baleiniers normands du X ${ }^{\mathrm{e}}$-XIII ${ }^{\mathrm{e}}$ siècle», Revue d'histoire économique et sociale, 42, 2, 1964, p. 147-161 et repris dans Nordica et Normannica, Paris, Société des études nordiques, 1997, p. 307-321.

Neveux, François, «Urbanisme au Moyen Âge dans quelques villes de Normandie», in L'architecture normande au Moyen Âge, Maylis Baylé (éd.), Condé-sur-Noireau, Éditions Corlet, 1997, I, p. 271-287.

Neveux, François, Bayeux et Lisieux, villes épiscopales de Normandie à la fin $d u$ Moyen Âge, Caen, Édition du Lys, 1996.

Pastoureau, Michel, «Les ménageries princières: du pouvoir au savoir $\left(\mathrm{XII}^{\mathrm{e}}-\mathrm{XVI}^{\mathrm{e}} \mathrm{s}\right.$.) », Micrologus, 18, 2008, p. 3-30.

Sivo, Francesca, «Valerio Massimo e Rodolfo Tortario», Invigilata Lucernis, 25, 2003, p. 251-278.

Sivo, Francesca, «Sul Testo del De Memorabilibus di Rodolfo Toirtario», Invigilata Lucernis, 35-36, 2014, p. 309-348.

VAllery-Radot, Jean, La cathédrale de Bayeux, Paris, Laurens, 1958 (2 édition).

\section{Annexe}

Raoul Tortaire, Épître IX, vers 137-316 ${ }^{32}$

Ac primum Cadomi sublimia tecta subivi,

Quae tanquam Pario marmore tota nitent; Innumeras merces illuc properando ferentes

$140 \quad$ Aspexi turbas undique confluere:

Sic examen apum dum nectit opus spetiosum,

Flava gerens caeris mella locanda novis,

Floribus excerptas pars advehit, altera caeras A fluvio sumptas pars quoque gestat aquas, Clausa cavis pars docta favos operatur in antris,

Arcet et infestas pars sibi bestiolas.

Diversae species holerum species et odorum

Et varii fuci lanea vestis ibi

Insuper et ${ }^{33}$ tenuis texturae copia lini,

32. Texte de l'édition de M. B. Ogle et Dorothy M. Schullian, avec renvois aux éditions partielles de Pluquet, 1829 et de Eugène de Certain, 1855.

33. Certain: est 
Multiplicis fili serica mollicies,

Cinnama, thura, piper, pomum, mel, caera, ciminum ${ }^{34}$

Setigerique sues lanigerique greges,

Exuviae pecudum multae pellesque ferarum

Nudatisque boum tergora visceribus

155 Nec plures mitis turmae deerant animantis,

Indomitis mixti cornipedes domiti,

Plurima cunctorum delectamenta ciborum

Potus quos fruges arbor et uva creant.

Vidi disparium facies habitusque virorum

160 Feminei sexus carbaseos apices:

Cum delata foro venalia tanta viderem

Non essent nummi quod mihi dirigui.

Et rex advenit, cuneo vallatus equestri,

Quem praecurrebant agmina miliciae,

165 Praebebat populo spectacula qui sibi grata:

Oderat Aethiopis verbera seva leo

Senos qui menses cum necdum parvus haberet,

Terrebat vulgus rictibus horrisonis:

Exulibus, miseris, prostratis ac spoliatis

Commodus et mitis dicitur esse leo;

Hinc maculis leopardus equo pulcherrimus atris

Colla vehebatur nexibus inplicitus:

Hunc creat in torva pardi genitura leena,

Velox inde feras saltibus exsuperat.

175 Concursu celeri properabat tota videre

Ore trucem lincem corpore plebs agilem

Cujus projecto deludit acumina vitro

Venator, raptis aufugiens catulis,

Denique sic visus acies transverberat ejus

180

Obvia quaque sibi vix quid ut officiat:

Curriculo Bachi linces potuere jugari

Ducitur ebrietas par quia seviciae;

Lubrica gibbosum ductabat turba camelum

Quem faleris comptus ${ }^{35}$ opido vitat equus:

185 Denique protenso caput est illi breve collo,

At gibbus dorso prominet in medio,

Quem tradit furiis ablata Venus diuturnis,

Bis centum fertur vivere solsticiis,

Quatriduam tolerare sitim, sed denuo quantum

Sufficeret fertur quatriduo bibere,

Fasci tollendo melior sit teste reciso,

\footnotetext{
34. Certain: cuminum

35. Certain: coopertum
} 
Marcia femineis bella placent gregibus.

$\mathrm{Nec}$ minus attonitam reddebat structio ${ }^{36}$ plebem

Collo procero cruribus inmodicis,

195 Qui sua non fovet ova, sed aggere pulveris abdit

Solaque vis animat ignea solis ea,

Sublevat a terra quem nequaquam sua pluma,

Ferrum ceu liquidos digerit iste cibos.

Purpura cum bisso regem vestibat et auro,

$200 \quad$ Ejus et ambibat pompa decens proceres.

Quicquid habet puchri, quicquid mundus ${ }^{37}$ preciosi,

Hac poeras turba cer..u. ${ }^{38}$ inspicere.

Solis inaurati clipei fulgore micabant

Splendebant tellus ${ }^{39}$ a quibus et fluvius

205 Qualiter Eolus cum, bella moventibus austris,

Nubes a quadro spiritus ${ }^{40}$ axe rapit,

Quarum fulmineas generat collisio flammas

Aer et arva ${ }^{41}$ micant nubigenis facibus.

Auro nobilium radiabat cultus equorum

210

A quorum fremitu tota tremebat humus,

Non aliter quam cum tonitrus quatit omnia sevus,

Concava riparum terraque mota tremit.

Musa situm Cadomi describere garrula gestit

De quo nil alias forsitan attigerat.

215 In ripa positum fluvii latus aspice castrum

Ad Boreum ${ }^{42}$ collis ediciore loco,

Prata virent latus ad Nothium longe secus amnem,

Per tria ducta fere milia lata parum;

Portus habet grandes sibi quas mittit mare naves,

Vix manat fluvius quo repetente sinu.

Terra ferax segetis, sed opacis indiga silvis,

Gallica nux, vitis ${ }^{43}$, ficus, oliva deest,

Insula diversis quam mercibus Anglia ditat

Et quaecumque ${ }^{44}$ mari cingitur occiduo.

225 Vespere jam subvectus equo feror hinc sitibundo, Inpotabilis est nam fluvius Cadomi,

Patronique mei comites qui primus agebat

\footnotetext{
36. Certain: struthio

37. Certain: habet

38. Certain: cernuus

39. Certain: turres

40. Certain: saepius

41. Certain: alta?

42. Certain: boream

43. Certain: vilis

44. Certain: quacumque
} 
Inpulsu, lautum ducor ad hospicium,

Transeo dum dubium dubitando secus mare rivum

230 Infertur praeceps in medium sonipes,

Exhauritur equis potantibus alveus amnis,

Limo ventre tenus sed meus obruitur.

Ast ego converso freni moderamine collo

Valdius infigor, conor ubi ire retro;

Anteriora cadunt, dum posteriora resurgunt,

Urgeo calcari, sentit id haud sonipes.

Navis in iratis pelagi sic fluctuat undis,

Puppis dum surgit prora profunda petit.

A sella caeno trepidus descendere conor,

Pendula jam nec equi girat habena caput;

Ergo volutabro multum versamur in illo

Clunes et pectus sparserat uda palus,

Angimur extensa nimium sonipes ego lucta,

Jamque fatigatus paene fatiscit equus.

245 Attollor panda minime rueram quia sella

Institerant pedibus vel quia scandilia;

Ilia quadrupedis rumpuntur agone paludis,

Ne garire putes, mortuus est sonipes,

Qui cursu rapido rapidos praecurreret Euros,

Leniter et cunctis planius iret equis.

Non inmunis ego sed aqua conspersus et ulva

Atque luto madidas sicco diu tunicas.

Hinc ad magnificae discumbo gausapa caenae,

Fercula seu calices enumerare piget;

255 Fertur inexpertis Babilonius archimagiris

Xerxes thesauros distribuisse suos,

Quosque parasse cibos reor haud magis illecebrosos

Rusticus exhibuit quam mihi rure coquus.

Orto sole die lunae discessimus inde,

Bajocense solum vespere nos recipit;

Passim dispersae nobis per litora villae

Exhibuere decem noctibus hospicia:

Infertur nostris pelagi vagus incola mensis,

Cujus non simplex aut sapor aut species;

265 Fluctibus atritos lego cum ductore lapillos,

Crure tenus limphas pervagor aequoreas;

Aequor id a nautis Platas fertur vocitari

Hiberno caete tempore quo capiunt.

Me praesente truci piscem clamore secuti,

Frustrati, casses nam deerant, redeunt;

Haud secus eductum densis de vepribus aprum

Turbaque venantum grexque canum sequitur, 
Personat a lituis altum nemus imaque vallis, $\mathrm{Heco}^{45}$ de scabris ingeminat scopulis.

275 Brumali coitus densantur tempore causa, Contrahit et plures femina sola mares

Una trahit tauros ut vacca petulca petulcos, Praecipitant validi viribus invalidos,

Gaudet littorei visis quibus accola pagi, Audet et irato bella parare salo.

$\mathrm{Hii}^{46}$ triplicant funes, hii torta cannabe casses

Texunt, hii calibem caute terunt hebetem.

Albicat eversus remorum verbere Nereus

Sulcat et aequoreum panda carina vadum.

285 Belua voce sono nautarumque impete vasto

Retibus occurrit dum tremefacta fugit,

Cernas ingentes proflantem ${ }^{47}$ naribus imbres

Caudae remigio mergere navigia.

Perfodiunt trifido pinguissima viscera ferro,

Ad litus trahitur saucia contiguum.

Hinc eo Bajocas ubi vidi culmina clara,

Turres excelsas aedis ${ }^{48}$ honorificae,

Intus quae saxo pulcre perfecta polito,

Exterius sculptis fulget imaginibus,

295 Multa metallorum locupletat quam variorum

Copia cum bisso, murice, vermiculo;

Ferrea sustentant argenti vincla coronam,

Alta quae dura sunt trabe fixa sude ${ }^{49}$.

Tota superficies auro vestita renidet,

$300 \quad$ Cinxit turritis quam faber aediculis,

Vix geminus templi paries capit hanc licet ampli,

Non aliam tanti ponderis esse reor.

Ingredior noti mediocria tecta sophistae

Gentatus ${ }^{50}$ quoniam vina peto fueram;

305 At sucus pomis datus est extortus acerbis,

Ori proposui dum reor esse merum;

Sed Bachus minime dominatur ${ }^{51}$ in hac regione,

Non ibi sunt linces, effera tigris abest,

Non valuere truces ejus propellere vires Jacto dente sati viperei populi,

45. Certain: echo

46. Certain: hi

47. Certain: perflantem

48. Certain: turris

49. Pluquet, Certain: Alte quae durae sunt clave fixa sudis

50. Pluquet: tentatus (var. jentatus)

51. Certain: donatur 
Viribus invictis ${ }^{52}$ nigros subjecit et Indos,

Nec sibi Normannos stravit adhuc rigidos.

Aspernor ciatum dum sencio non fore vinum,

Fingo bibisse tamen labraque sicco mea;

«Cur propinasti, serve, venena mihi?»

\section{Traduction}

D’abord j'ai déambulé sous les hauts toits de Caen, qui resplendissent tous, tel le marbre de Paros. J'ai aperçu des foules affluer de toutes parts, apportant là en toute hâte d'innombrables marchandises, tel un essaim d'abeilles tissant son merveilleux ouvrage et portant le miel doré pour le disposer dans de nouvelles alvéoles de cire: un groupe transporte les cires arrachées aux fleurs, un autre apporte l'eau puisée dans le fleuve, un autre, enfermé dans les ruches et expert dans la fabrication des rayons, en produisent de nouveaux et un dernier repousse loin des ruches les insectes nuisibles.

Il y avait là diverses espèces de légumes, diverses sortes d'odeurs ainsi que des vêtements de laine aux teintures variées. On trouvait, en plus, une abondance de toiles de lin d'un fin tissage, de la soie souple aux fils innombrables, de la cannelle, de l'encens, du poivre, des fruits, du miel, de la cire, du cumin, des sangliers hérissés de poils et des troupeaux de moutons, quantité de peaux d'animaux et de dépouilles de bêtes sauvages ainsi que des carcasses de bœufs débarrassés des viscères. Ne manquaient pas non plus les troupes nombreuses d'animaux vivants et dociles, les chevaux dressés mêlés aux chevaux sauvages, les séductions sans fin de tous les aliments ainsi que les boissons que l'on extrait des fruits, des arbres et de la vigne. J'ai vu les apparences diverses et les tenues différentes des hommes ainsi que les coiffes de lin des femmes. En contemplant tant de produits apportés sur la place pour y être vendus, je demeurai immobile, vu que je n'avais pas d'argent.

Le roi arriva, entouré d'un contingent de chevaliers, précédés d'une avantgarde de soldats. Il offrait des spectacles qui faisaient le plaisir du peuple. Un lion, qui n'avait pas encore six mois, n'appréciait guère les coups cruels d'un éthiopien et terrifiait la foule par ses rugissements terrifiants. Le lion est, dit-on, doux et bienveillant envers les exilés, les miséreux, les malheureux et les gens que l'on a dépouillés. Ensuite un magnifique léopard aux taches sombres monté sur un cheval, se cramponnait à sa crinière. Il est le produit d'une union anormale entre une lionne et un pard; de ce fait, il est rapide et surpasse les autres bêtes sauvages par ses bonds. Toute la foule se hâtait en accourant rapidement d'aller voir un lynx au corps agile et à la gueule terrifiante, dont le chasseur, protégé par une vitre, se joue de ses griffes, alors qu'il s'enfuit après avoir emporté ses petits. La pénétration de son regard détecte tout ce qui vient à sa rencontre, de

52. Certain : invicti 
sorte que rien ne peut lui faire obstacle. Les lynx ont pu faire partie du cortège de Bacchus, car l'ébriété est associée à la cruauté. Un curieux groupe conduisait un dromadaire avec sa bosse, animal qu'évite absolument le cheval, orné de phalères. Il a une tête courte avec un long cou, mais la bosse domine au milieu de son dos. Cet animal qui accompagne Vénus, délivrée de ses longs délires, vit, dit-on, deux cents solstices. On dit aussi qu'il supporte une soif de quatre jours, mais qu'il boit à satiété de nouveau le quatrième jour. Une fois castré, il serait un meilleur porteur de fardeau, mais c'est aux troupeaux de dromadaires femelles que plaisent les guerres de Mars. Une autruche, au long cou et aux pattes démesurées, provoquait tout autant l'étonnement de la foule: elle ne couve pas ses œufs, mais les cache sous un tas de poussière et c'est la puissance de feu solaire qui leur donne vie. Son plumage ne lui permet pas de quitter le sol et elle digère le fer aussi bien que les nourritures liquides.

Le roi était revêtu d'un habit pourpre et d'une tunique de lin fin rehaussé d'or et il était entouré d'un cortège harmonieux de grands personnages. En tendant la tête (?) tu pouvais contempler tout ce que le monde avait de beau et de précieux. L'or des boucliers renvoyait les rayons du soleil et faisait briller la terre et le fleuve, tel Éole, lorsque son souffle, au moment où les vents du sud relancent les guerres, fait disparaitre des quatre régions du ciel les nuages, dont la collision provoque le feu de la foudre, et que l'air et l'éther sont alors illuminés par les torches engendrées par les nuages. Les parures d'or des nobles chevaux étincelaient, et leurs hennissements faisaient trembler toute la terre; c'est ainsi que tremblent la terre ébranlée et le creux des rivages, quand le tonnerre en fureur frappe toutes choses.

La Muse bavarde brûle de décrire le site de Caen, dont il n'a été sans doute question nulle part ailleurs. Vois le château situé sur la rive, près du fleuve, au nord à l'endroit le plus élevé de la colline. Au sud, des prés verts de faible largeur s'étendent sur près de trois milles, tout près de la rivière. Le port abrite des navires imposants, que la mer lui envoie, là où le fleuve a du mal à s'écouler en raison de la mer qui remonte dans l'estuaire. La terre est riche en moissons, mais pauvre en forêts épaisses. Font défaut la noix gauloise, la vigne, le figuier et l'olivier. L'île d'Angleterre, qui est entourée par la mer occidentale, l'enrichit de diverses marchandises. Vers le soir, je quitte l'endroit, déjà monté sur mon cheval assoiffé, car l'eau du fleuve de Caen n'est pas potable. À l'initiative de mon protecteur qui en tête faisait avancer mes compagnons, je suis conduit vers une auberge somptueuse. Tandis que je traverse avec hésitation un ruisseau dangereux situé près de la mer, mon cheval se laisse emporter au beau milieu. Le lit du ruisseau se vide tandis que les chevaux y boivent, mais mon cheval s'enfonce dans la vase jusqu'au ventre. Alors moi, bien que j'aie fait tourner le cou de l'animal à l'aide du mors, je m'enfonce davantage là où j'essaie de faire marche arrière. Les antérieurs de mon cheval s'enfoncent, en relevant les postérieurs. Je m'emploie à jouer des éperons, mais l'animal ne réagit pas. Tel un navire ballotté sur les flots déchaînés, tandis que la poupe s'élève, la proue plonge dans les profondeurs. Tout tremblant, j'essaie de descendre de ma selle dans la boue, mais la bride qui désormais pend ne peut plus faire tourner la tête du cheval; nous demeurons 
enfoncés dans cette vase, alors que le marais plein d'eau avait recouvert la croupe et la poitrine de mon cheval. Nous sommes dans une grande angoisse, ma monture et moi, tandis que nous livrons cette lutte acharnée, mais le cheval épuisé succombe presque de fatigue. Je me redresse, car je n'avais pas quitté la selle courbe, les étriers toujours fixés à mes pieds. Les flancs du quadrupède se rompent sous la pression du marais. Ne songe pas à plaisanter, mon cheval est mort, lui qui par sa course rapide était capable de devancer les rapides vents du sud et d'aller d'une allure tranquille et plus aisée que tous les autres chevaux. Moi, je ne m'en sors pas indemne, mais couvert d'eau et de boue, et la vase séchée a longtemps souillé mes vêtements trempés. Ensuite je m'étends sur la couverture d'une resplendissante salle à manger et il m'en coûte d'énumérer les plats et les coupes. On rapporte que Xerxès le babylonien avait distribué ses trésors à ses cuisiniers incapables et les mets qu'il avait préparés, n'étaient, je pense, guère plus séduisants, que ceux que mon paysan cuisinier m'a offerts à la campagne.

Le lundi, au lever du soleil, nous quittons les lieux. Le soir, c'est le pays $\mathrm{du}$ Bessin qui nous accueillit. Des demeures qui se trouvaient tout au long du rivage nous offrirent l'hospitalité les dix nuits de notre périple. On apporte sur nos tables des êtres vivants qui errent au sein des flots, d'espèces et de saveurs différentes. En compagnie de mon guide je ramasse des galets polis par les flots et je me promène, jambes dans l'eau, à travers des mares, que les marins appellent des "plates», là où, en hiver, ils piègent des baleines. En ma présence, après avoir poursuivi un de ces poissons en poussant des cris effroyables, ils rentrent déçus, car leurs filets de pêche n'ont pas tenu. C'est ainsi que la troupe des chasseurs et la meute des chiens poursuivent le sanglier qui a jailli des broussailles épaisses, et que la haute forêt et la profonde vallée retentissent du son des cors que renvoient en écho les rochers hérissés. En hiver, les baleines se multiplient en raison des accouplements, une seule femelle s'unit à un grand nombre de mâles, comme une seule vache à cornes attire les taureaux à cornes, les plus vigoureux écartant par leur puissance les plus faibles.

Dès qu'ils ont aperçu les baleines, les gens proches du rivage se réjouissent et n'ont pas peur de préparer l'affrontement dans une mer déchaînée. Les uns triplent les câbles, d'autres tissent des filets avec du chanvre enroulé, d'autres aiguisent avec soin les harpons émoussés; alors Nérée blanchit sous les coups portés par les rameurs et la carène courbe fend les flots de la mer. La bête contrainte par les cris, les bruits et les assauts démesurés des marins se jette dans les filets dans sa fuite épouvantée. Tu aurais vu la baleine, qui soufflait par ses narines d'immenses jets d'eau, faire chavirer les embarcations par le mouvement de sa queue. Les marins enfoncent dans ses flancs couverts d'une épaisse peau graisseuse le fer à la triple pointe et la baleine blessée est tirée jusqu'au rivage tout proche.

De là, je vais à Bayeux, où j'ai vu des édifices somptueux, les hautes tours d'une église vénérable, qui parfaitement achevée resplendit à l'intérieur par ses pierres parfaitement polies et à l'extérieur par ses représentations sculptées. Elle s'enrichit d'une abondance d'objets de divers métaux ainsi que d'une toile de lin, de couleur pourpre et vermeil. Des chaînes en fer, fixées tout en haut à une poutre par une solide attache, supportent une couronne d'argent. Celle-ci est 
entièrement recouverte d'or étincelant et le forgeron a disposé tout autour des édicules en forme de tours. Elle n'a pas, à mon avis, son pareil en poids et trouve difficilement place entre les deux murs de l'église, malgré ses grandes dimensions.

J'entre dans la modeste demeure d'un orateur connu et comme j'en avais envie, je demande du vin. Mais on m'a apporté un breuvage qui est un extrait de pommes acides: je l'ai porté à mes lèvres croyant que c'était du vin; mais Bacchus n'offre guère ses dons dans ce pays, où il n'y a ni lynx ni tigre féroce. Les peuples vipérins nés de la dent du dragon n'ont pas réussi à repousser les forces sauvages de Bacchus, qui a également soumis les noirs indiens à sa puissance invincible, mais il n'a pas encore terrassé ces inflexibles Normands. Je repousse la coupe en sentant que ce n'était pas du vin; je feignis cependant avoir bu et m'essuyai les lèvres; je rends la coupe au garçon auquel je murmure en me penchant à son oreille: «Pourquoi, garçon, m’as-tu proposé ce poison?». 